\title{
Hábitos nutricionales y estados emocionales en tiempos de COVID-19
}

\author{
Nutritional habits and emotional states in COVID-19 time
}

Yanina Milagros Torres Huamani $\unrhd$

Universidad Le Cordon Bleu. Lima, Perú

Recibido: 09/04/2021 Revisado: 21/05/2021_Aceptado: 07/07/2021 Publicado: 31/10/2021

\section{RESUMEN}

El estilo de vida saludable reduce las posibilidades de contraer enfermedades degenerativas, sin embargo; durante la pandemia COVID-19 se presentaron dificultades para su constancia. El sobrepeso y la obesidad se asocian a conductas de alimentación que afectan la salud, y en consecuencia alteran las respuestas inmunitarias que debilitan las defensas, aumentando la probabilidad a contraer esta enfermedad. El objetivo del presente artículo fue determinar las consecuencias nutricionales y alteraciones emocionales ocasionadas por el sedentarismo provocado por el SARS-CoV-2. Se realizó una búsqueda sistemática de publicaciones en las bases de datos de SciELO, Dialnet, Science Direct, Pub Med, la Organización Mundial de la Salud (OMS), Association of Obesity, British Medical Journal, American Society for Microbiology y Infectious Diseases Society of America, sobre consecuencias y causas de la obesidad y sus agravantes. Paralelamente, se realizó una encuesta a 51 personas para identificar las condiciones a las que se enfrentaron durante el confinamiento. Los encuestados declaran haber experimentado cambios significativos en su día a día, principalmente al momento de adquirir alimentos, es decir, por la disposición de alimentos saludables, asimismo, la inactividad física debido al aislamiento y un deterioro en su estado físico. El tener un estilo de vida saludable es una prioridad, para prevenir enfermedades futuras. Este trabajo puede servir como un referente para futuras investigaciones que intenten identificar aquellos factores que repercuten los estilos de vida a causa del confinamiento provocado por la COVID-19. Palabras clave: COVID-19 estados emocionales, hábitos alimentarios, obesidad, sobrepeso, microbiota intestinal.

\section{ABSTRACT}

Healthy lifestyles reduce the likelihood of contracting degenerative diseases, however; during the COVID-19 pandemic, there were difficulties in sustaining them. Overweight and obesity are associated with eating behaviors that affect health and consequently alter immune responses that weaken defenses, increasing the likelihood of contracting this disease. This article 
aimed to determine the nutritional and emotional consequences of sedentary lifestyles caused by SARS-CoV-2. A systematic search was conducted of publications in the databases of SciELO, Dialnet, Science Direct, Pub Med, the World Health Organization (WHO), Association of Obesity, British Medical Journal, American Society for Microbiology and Infectious Diseases Society of America, on the consequences and causes of obesity and its aggravating factors. In parallel, 51 people were surveyed to identify the conditions they faced during confinement. Respondents reported significant changes in their daily lives, mainly in terms of food shopping, i.e. the availability of healthy food, physical inactivity due to isolation, and a deterioration in their physical condition. A healthy lifestyle is a priority to prevent future illnesses. This work can serve as a reference for future research to try to identify those factors that impact lifestyles due to COVID-19 confinement.

Keywords: Covid-19, emotional states, eating habits, obesity, overweight, gut microbiota.

\section{INTRODUCCIÓN}

La Organización Mundial de la Salud (OMS) declaró la obesidad como una enfermedad presente en todo el mundo, considerada la pandemia del siglo XXI. Las mayores prevalencias ocurren en la población adulta y seguida por el grupo de juvenil (Gutiérrez-Fisac et al., 2003). Su presencia se relaciona a enfermedades como la diabetes mellitus 2 , la hipertensión arterial, las dislipidemias y algunos tipos de cánceres, siendo un factor de riesgo importante (Pajuelo-Ramírez, 2017).

El estilo de vida no saludable, como el sedentarismo y los malos hábitos nutricionales son cada vez más habituales y con una tendencia a diseminarse en el futuro, lo cual hace que el panorama de la obesidad constituya un reto para la salud pública, principalmente en países desarrollados. Asimismo, tiene su origen en una interacción genética, ambiental y conductual, siendo más importante la parte ambiental y conductual, que se establece por un desequilibrio entre la ingesta, los malos hábitos nutricionales y el gasto energético (Quirantes, et al., 2009).
Tras el confinamiento debido a la llegada de la COVID-19, se hizo visible la importancia de contar con un buen estado nutricional, dado que entre los grupos vulnerables se encuentran las personas con sobrepeso, obesidad, hipertensión, entre otros padecimientos. Se sabe que el contar con una buena actividad física, una dieta correcta, considerando la selección y preparación de los alimentos, la frecuencia y cantidades de consumo, son acciones fundamentales para paliar las consecuencias de los cambios del estilo de vida que implica el periodo de aislamiento o cuarentena y la incertidumbre que genera (Salle \& Ayala-moreno, 2020).

La federación mundial de la obesidad señaló que las afecciones relacionadas con la obesidad parecen empeorar el efecto de la COVID-19, de igual manera profesionales de la salud enfatizan tener especial cuidado en personas con un IMC de 40 o más, para evitar infecciones primordialmente para personas con obesidad. El distanciamiento físico necesario para poder controlar la diseminación del coronavirus, para muchas personas ha sido un generador de 
estrés, ansiedad y agresividad, mientras que para otras ha sido un espacio de análisis sobre su estilo y forma de vida (Rodríguez, 2020)

Esta propagación ha significado un cambio en las tendencias y los hábitos alimentarios que probablemente continúen después de las medidas que se han tomado, dentro de los cuales destaca la obesidad, la seguridad alimentaria, disponibilidad, acceso, así como las implicaciones de la ansiedad, estrés emocional sobre el consumo de alimentos (Salle \& Ayala, 2020). El objetivo del presente artículo fue determinar las consecuencias nutricionales y alteraciones emocionales ocasionadas por el sedentarismo provocado por el SARS-CoV-2.

\section{MATERIALES Y MÉTODOS}

\section{Investigación bibliográfica}

Se realizó una búsqueda sistemática de información publicada en los últimos 17 años en las bases de datos SciELO, Dialnet, Science Direct, Pub Med, la Organización Mundial de la Salud (OMS) Association of Obesity, British Medical Journal, American Society for Microbiology y Infectious Diseases Society of America, para lo cual se empleó los siguientes términos de búsqueda: Obesidad, COVID-19, hábitos nutricionales, sedentarismo, sobrepeso, estados emocionales, horas de sueño y microbiota intestinal

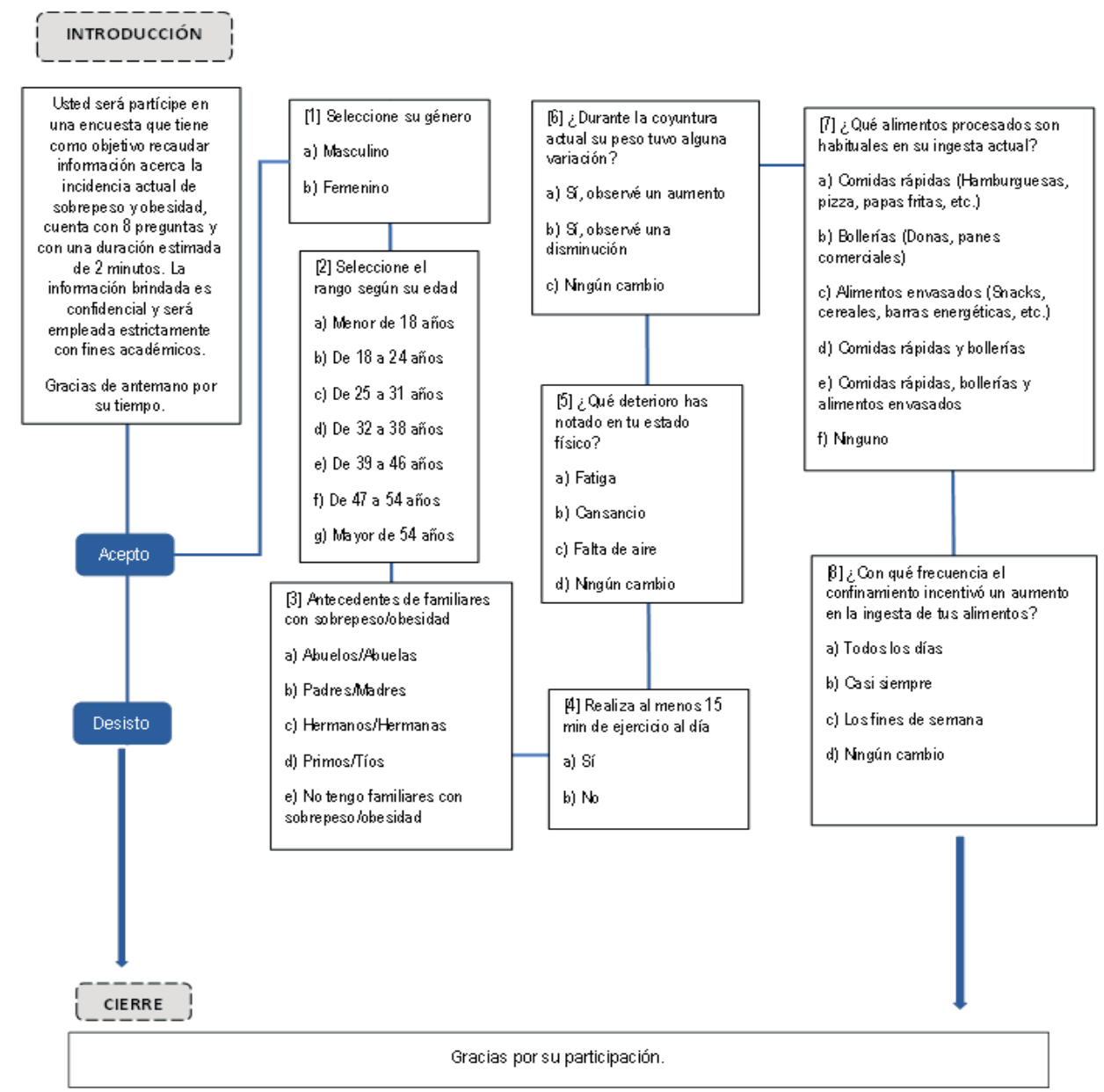

Figura 1. Estructura de encuesta aplicada con el fin de recaudar información acerca la incidencia actual de sobrepeso y obesidad. Preguntas realizas en la encuesta virtual. 


\section{Encuestas de opinión}

Se aplicó una encuesta compuesta por 8 preguntas, a 51 personas (26 varones y 25 mujeres), de 18 a 46 años. Esta fue realizada con la finalidad de identificar las condiciones en las que se encuentran durante la coyuntura del COVID-19 y recaudar información acerca la incidencia actual vinculadas al sobrepeso y obesidad (Figura 1). La encuesta fue diseñada en Google forms y compartida a través de redes sociales.

Tabla 1. Clasificación de la OMS del Sobrepeso y Obesidad según el IMC

\begin{tabular}{lcc}
\hline Clasificación & $\begin{array}{c}\text { IMC } \\
(\mathbf{k g} / \mathbf{m 2})\end{array}$ & Riesgo en la Salud \\
\hline Sobrepeso & $\geq 25.0$ & Alto \\
Pre Obesidad & $25.0-29.9$ & Alto \\
Obesidad & $\geq 30.0$ & Alto \\
Obesidad Grado I & $30.0-34.9$ & Alto Moderado \\
Obesidad Grado II & $35.0-39.9$ & Alto Severo \\
Obesidad Grado III (Obesidad mórbida) & $\geq 40.0$ & Extremadamente Alto \\
\hline
\end{tabular}

Fuente: Adaptado OMS (Organización Mundial de la Salud)

\section{Sobrepeso y obesidad como factor de riesgo}

La prevalencia del sobrepeso y la obesidad ha aumentado dramáticamente, hasta hace pocos años no se consideraba una enfermedad más bien una condición predisponente para el desarrollo de otros padecimientos. Sin embargo, la tendencia actual la reconoce como una enfermedad crónica (Julia et al., 2016). La cual es inducida por factores genéticos, hormonales, ambientales, emocionales y culturales, teniendo mayor riesgo de desarrollar muchas afecciones médicas graves, incluida la

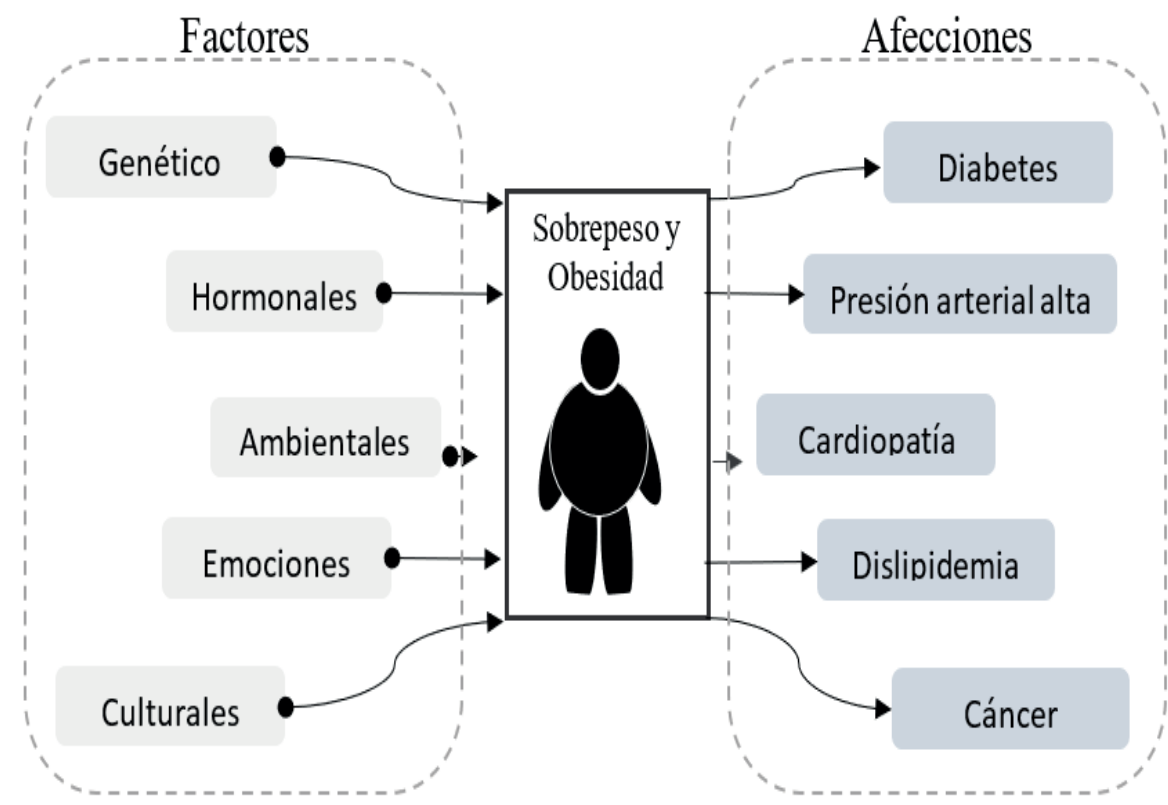

Figura 2. Factores de riesgo del Sobrepeso y Obesidad 
diabetes tipo 2, presión arterial alta, cardiopatía y dislipidemia, también está asociado a un mayor riesgo de diversos tipos de cánceres (Figura 2). La evidencia de que la obesidad es un factor de riesgo para severidad por SARSCoV-2 que está aún en aumento (Tenorio \& Hurtado, 2020), teniendo un papel importante en la patogénesis de la infección de la misma. De hecho las personas con obesidad en todo el mundo ya tienen un alto riesgo de sufrir complicaciones graves de COVID-19 (Ryan et al., 2020).

\section{Sobrepeso y obesidad}

El sobrepeso y obesidad presenta una tendencia hacia el incremento que afecta la calidad de vida de la población en general. La presencia de ambas afecciones no solo tiene impacto en la mortalidad, sino que también es un factor predisponente a la infección del SARS-CoV-2. La clasificación del sobrepeso

y obesidad propuesta por la OMS está basada en el Índice de Masa Corporal (IMC), el cual corresponde a la relación entre el peso expresado en kilos y el cuadrado de la estatura, expresada en metros. De tal manera, las personas cuyo cálculo de IMC sea igual o superior a $30 \mathrm{~kg} / \mathrm{m} 2$ se consideran obesas (Tabla 2).

\section{Estilo de vida asociado al sobrepeso y obe- sidad}

Es evidente que el llamado a realizar cambios en el estilo de vida es una prioridad en el tema de salud mundial, lamentablemente el interés actual por la alimentación cada día ha generado un entorno de errores o engaños, con el fin de desestimar la importancia de alimentos en nuestra dieta (Erem, 2014). Existen diversos factores que inducen al sobrepeso y obesidad, entre los que destacan, la mala ali- mentación, seguida por el sedentarismo y la falta de acceso a alimentos nutritivos. Durante la coyuntura actual se manifestó un incremento en el consumo de alimentos y bebidas procesadas y ultra procesadas, caracterizadas por una calidad nutricional deficiente; que fueron empleadas como reemplazo a las comidas caseras, aumentando el riesgo de estos estados degenerativos. Por otro lado, la actividad física se vincula de manera reiterativa a los hábitos de vida saludable para mejorar la salud física y mental de las personas (Pérez, 2014) siendo una importante iniciativa, ya que numerosos estudios han demostrado los efectos beneficiosos en la disminución del peso y la masa grasa en los individuos, por consiguiente la inactividad en este contexto se ha convertido en un factor de riesgo para la salud pública. El sedentarismo en consecuencia, multiplica la morbimortalidad.

Uno de los cambios comportamentales en la sociedad ha sido la disminución de las horas destinadas al sueño nocturno, por ello es importante resaltar que, la cantidad, calidad y horario de sueño, siendo un factor relacionado a la obesidad (Chamorro et al., 2011). Durante el sueño se ahorra y se almacena energía, se reducen los procesos digestivos y se llevan a cabo procesos de reparación celular, estos estados que se alternan diariamente, tan distintos en requerimientos energéticos que afectan a todos los tejidos y órganos del cuerpo y son coordinados por el reloj biológico, que transmite señales temporales a todo el cuerpo. La utilización diaria de aparatos electrónicos como celulares, tablet, laptop, televisor, entre otros, en periodos prolongados ocasiona un retraso en el inicio del sueño y reducción del mismo; el inadecuado patrón de sueño podría contribuir a alteraciones metabólicas tempranas, conducentes a mayor ganancia de peso y 
riesgo de obesidad por parte de todos los grupos etarios y segmentos de la sociedad, que podría constituir un factor con impacto potencial a nivel de salud pública en la prevención de la obesidad (Chamorro et al., 2011). Durante la pandemia las personas compensaban las actividades sociales a través de interacción en redes sociales, reuniones en línea, videojuegos, entre otros, propiciando a un desorden del sueño, cansancio visual y sedentarismo.

\section{El papel de la obesidad en la COVID-19}

La OMS y la federación mundial de la obesidad declaran la obesidad como una enfermedad presente en todo el mundo, que caracteriza a individuos con un IMC $>40$ y 42 para hombres y mujeres, respectivamente. Esta enfermedad origina alteraciones en la respuesta inmunitaria generando un proceso inflamatorio que suele ser crónico (Blancas et al., 2010), esta respuesta hiperactiva del sistema inmunológico ante la COVID-19 hace que sea difícil combatir el virus (Figura 3).

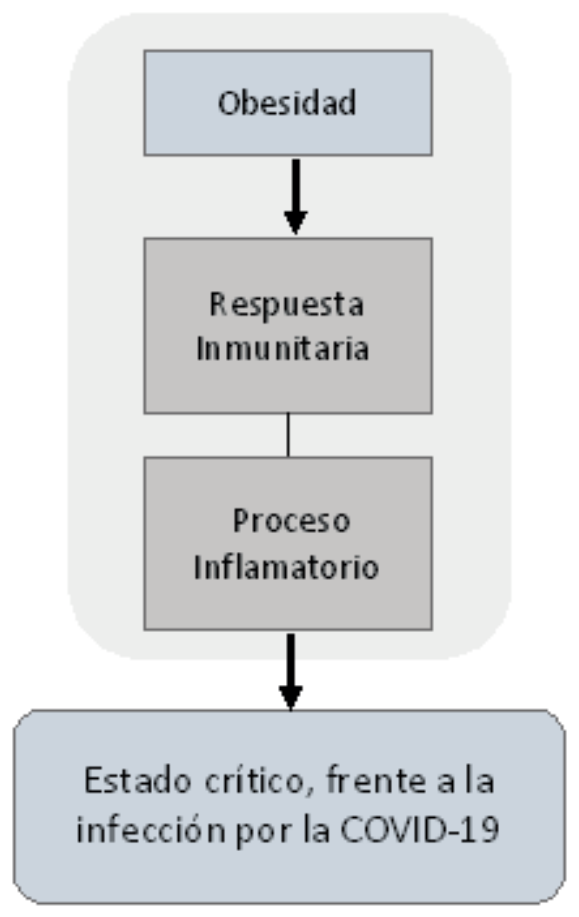

Figura 3. Papel de la obesidad en la probabilidad de infección por COVID-19
Al inicio de la pandemia fue una variable omitida, en la mayoría de los casos por las mismas personas que padecen de obesidad. Al respecto, se han publicado estudios que refieren que los sujetos con obesidad tienen mayor riesgo de desarrollar una enfermedad más grave por este virus. Klang et al. (2020) analizaron retrospectivamente los datos obtenidos de pacientes con COVID-19, que fueron hospitalizados en un sistema hospitalario académico en la ciudad de Nueva York. Los datos incluyeron datos demográficos, comorbilidades, IMC y tabaquismo. Los grupos de obesidad incluyeron los siguientes: IMC de 30 a $<40 \mathrm{~kg} / \mathrm{m} 2$ e IMC $\geq 40$ $\mathrm{kg} / \mathrm{m} \mathrm{2}$, siendo predisponentes a fallecer.

Kalligeros et al. (2020) también realizaron otro estudio retrospectivo con 103 pacientes para relacionar el impacto del IMC y la gravedad frente a la COVID-19. Este estudio concluye que la obesidad grave IMC $\geq$ 35 kg.m-2 está asociado con el ingreso en la unidad de cuidados críticos (UCI). Asimismo, un IMC $\geq 30 \mathrm{~kg} \cdot \mathrm{m}-2$ con antecedentes de daño cardiaco está asociado a riegos del uso de ventilación mecánica intensiva (VMI).

\section{Microbiota intestinal y su influencia en la COVID-19}

La COVID-19 es principalmente una enfermedad respiratoria, sin embargo; existe una creciente evidencia que sugiere que el tracto gastrointestinal está involucrado en esta enfermedad. Por lo tanto, las afecciones crónicas como la obesidad se asocian con un microbiota intestinal alterada (disbiosis) (Kim, 2021). Las bacterias que conforman la microbiota son capaces de modular la respuesta inflamatoria, por lo que su alteración afectaría de forma negativa y podría provocar una respuesta inmune agresiva que agravaría 
las consecuencias de este virus (Yeoh et al., 2021). Un estudio reciente (Gu et al., 2020), mostraron una reducción significativa en la diversidad bacteriana de la microbiota intestinal recolectada en pacientes con COVID-19 a comparación de las obtenidas de controles sanos. Estos cambios en la microbiota intestinal generalmente se consideran signos de disbiosis y un intestino no saludable (Kim, 2021).

Es fundamental tener una microbiota balanceada para evitar la disbiosis, donde se contemple una buena cantidad de Bifidobacterium, Faecalibacterium prausnitzii y Eubacterium rectale, quienes podrían protegernos frente a una infección severa por la COVID-19 (Yeoh et al., 2021).

\section{Estados emocionales asociados al sobrepeso} y obesidad

Las emociones afectan la respuesta alimentaria a lo largo de todo el proceso de ingestión, la motivación del comer, las respuestas afectivas a los alimentos, la elección, la masticación y velocidad con que se ingieren (Palomino-Pérez, 2020). Asimismo, las emociones poseen características placenteras como también las que presentan displacer, es decir las negativas, las que hacen que aumenten la ingesta de alimentos, por ejemplo: Estrés, la frustración, ira, ansiedad, el miedo, entre otros. Cabe señalar que la ira es una emoción que influye de manera importante en el comer por atracones (Palomino-Pérez, 2020), por otro lado, la ansiedad más

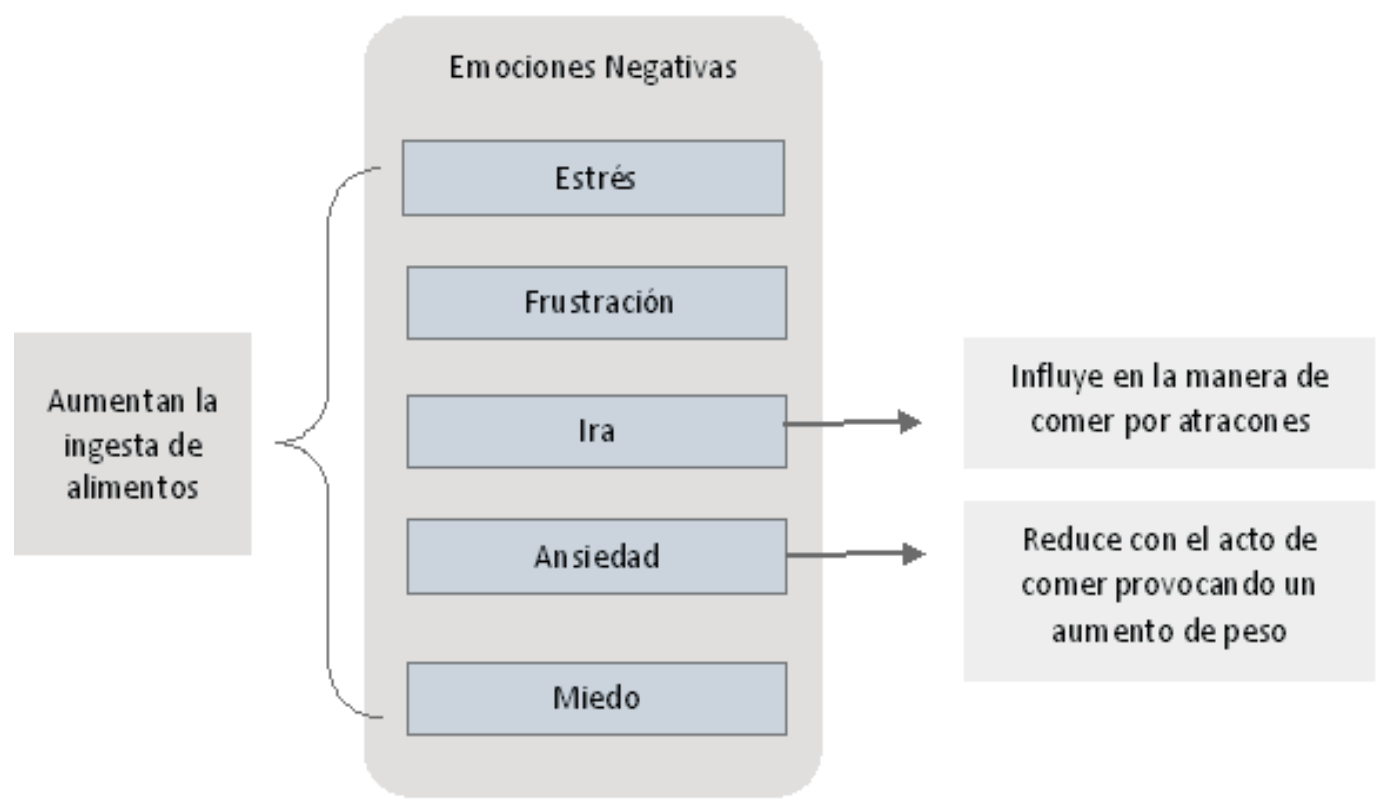

Figura 4. Emociones negativas que afectan la forma de alimentarnos

relacionada con la obesidad es la ansiedad generalizada, aquella que está presente a niveles no muy elevados y se reduce con el acto de comer, con lo que provoca un aumento de peso. (Guzmán, 2010), véase (Figura 4).

\section{RESULTADOS Y DISCUSIÓN}

De acuerdo a los resultados de la encuesta aplicada, 19 de los encuestados refieren no tener familiares con presencia de sobrepeso y obesidad, 11 poseen antecedentes por parte de los padres de familia, 8 por parte 
de abuelos y abuelas, 5 por primos y tíos y 8 por parte de hermanos. Asimismo, el $70 \%$ manifiesta realizar actividad física al menos 15 minutos y un $30 \%$ no lo hace; por otro lado, el $52 \%$ refiere tener haber presentado un deterioro en su estado físico, el $30 \%$ expresan que no observaron ningún cambio, $9 \%$ por tema de la fatiga y un $9 \%$ por falta de aire. El $61 \%$ experimentó un aumento de: peso a raíz de basar su alimentación en alimentos procesados y comidas rápidas como Hamburguesa, pizza, papas fritas, entre otros, y el $39 \%$ no manifiesta ningún cambio. El $37 \%$ tuvo una ingesta excesiva de alimentos de baja calidad nutricional cada fin de semana, $32 \%$ expresa no tener ningún cambio al respecto, el $31 \%$ casi siempre, y el $10 \%$ refiere estar siendo afectado todos los días. (Tabla 2).

Tabla 2. Preguntas resaltantes y resultados de la encuesta aplicada

Tabla 2. Preguntas resaltantes y resultados de la encuesta aplicada

\begin{tabular}{|c|c|}
\hline \multicolumn{2}{|c|}{ Resultados de la Encuesta aplicada } \\
\hline Antecedentes familiares con sobrepeso/obesidad & $\begin{array}{l}\text { Realiza actividad física al menos } 15 \\
\text { minutos (\%) }\end{array}$ \\
\hline \multicolumn{2}{|l|}{19 - No tienen Familiares con sobrepeso y obesidad } \\
\hline 11- Por parte de los padres & 70 - Lo hace \\
\hline 8 - Por parte de abuelos y abuelas & 30 - No lo hace \\
\hline \multicolumn{2}{|l|}{8 - Por parte de hermanos } \\
\hline \multicolumn{2}{|l|}{5 - Por parte de primos y tíos } \\
\hline Has notado un deterioro en tu estado físico (\%) & $\begin{array}{l}\text { Durante la coyuntura actual su peso tuvo } \\
\text { alguna variación (\%) }\end{array}$ \\
\hline \multicolumn{2}{|l|}{52 - Presenta un deterioro en su estado físico } \\
\hline 30 - No observa cambio & 61 - Aumento de peso \\
\hline 9 - Fatiga & 39-Ningún cambio \\
\hline \multicolumn{2}{|l|}{9 - Falta de aire } \\
\hline excesiva de tus alimentos (\%) & \\
\hline \multicolumn{2}{|l|}{37 - Ingesta excesiva } \\
\hline \multicolumn{2}{|l|}{32 - Ningún cambio } \\
\hline \multicolumn{2}{|l|}{31 - Casi siempre } \\
\hline 10 - Todos los días & \\
\hline
\end{tabular}


Los participantes declaran haber experimentado cambios significativos en su día a día, principalmente al momento de adquirir alimentos, especialmente alimentos como comidas rápidas siendo de poco valor nutritivo. Actualmente la mayoría de la población lo hace a través de aplicaciones de delivery, lo que conlleva a una inactividad física aspecto que se debe que tomar en cuenta.

Las medidas de confinamiento decretadas a causa de la COVID-19, así como los temores individuales asociados a esta enfermedad, han agravado condiciones preexistentes de sobrepeso y obesidad, y en otros casos se ha propiciado su aparición dada la disminución de actividad física, hábitos alimenticios, cambios emocionales y las horas de sueño, es decir, son afectados por repercusiones de su estilo de vida deficiente.

\section{CONCLUSIONES}

Antes de que llegase el SARS-CoV-2, el sobrepeso y obesidad era una pandemia que cada vez afecta a los diferentes grupos etarios y esto se fue agravando con la pandemia, siendo uno de los factores que dificultaba el pronóstico durante la infección por la COVID-19.
Actualmente las conductas nutricionales, los estados emocionales y el sedentarismo influyen significativamente en la salud de la población, asimismo; se enfatiza factores importantes como, ansiedad, la cantidad y calidad de sueño especialmente en la incertidumbre del confinamiento a causa de la llegada de la COVID-19, propiciando un riesgo de padecer sobrepeso y obesidad, además estos estados negativos también es un factor predisponente de infección por este virus. Dentro del análisis expuesto.

Los participantes de la encuesta expresan haber tenido cambios significativos durante el confinamiento, por el consumo excesivo de alimentos poco nutritivos, deterioro en su estado físico y por ende un aumento en peso. Así mismo, fueron afectados emocionalmente debido a un deterioro de su estado físico, como la fatiga o falta de aire que induce a una disminución de la actividad física.

\section{Agradecimientos}

La autora reconoce y agradece la generosidad de las personas que participaron en la encuesta, al Dr. Oscar Benjamín Jordán Suárez por su colaboración y asesoramiento y finalmente a los artículos de revisión que ayudaron en la investigación.

\section{REFERENCIAS BIBLIOGRÁFICAS}

Blancas-Flores, G., Almanza-Pérez, J. C., López-Roa, R. I., Alarcón-Aguilar, F. J., García-Macedo, R., \& Cruz, M. (2010). La obesidad como un proceso inflamatorio. Bol. Méd. Hosp. Infant. Méx, 67(2), 88-97.

Chamorro, R. A., Reyes, S. C., Ponce, R., \& Peirano, P. D. (2011). La reducción del sueño como factor de riesgo para obesidad. 932-940.

Erem, S. Y. (2014). Cómo lograr una vida saludable. Anales Venezolanos de Nutricion, 27(1), 129-142. 
Gu, S., Chen, Y., Wu, Z., Chen, Y., Gao, H., Lv, L., Guo, F., Zhang, X., Luo, R., Huang, C., Lu, H., Zheng, B., Zhang, J., Yan, R., Zhang, H., Jiang, H., Xu, Q., Guo, J., Gong, Y., ... Li, L. (2020). Alterations of the Gut Microbiota in Patients With Coronavirus Disease 2019 or H1N1 Influenza. Clinical Infectious Diseases, 71(10), 2669-2678. https://doi.org/10.1093/cid/ciaa709

Gutiérrez-Fisac, J. L., Regidor, E., López García, E., Banegas Banegas, J. R., \& Rodríguez Artalejo, F. (2003). [The obesity epidemic and related factors: the case of Spain]. Cadernos de Saude Publica, 19 Suppl 1, S101-10. http://www.ncbi.nlm.nih.gov/pubmed/12886440

Guzmán SRME, del Castillo AA, G. M. (2010). Factores psicosociales asociados al paciente con obesidad. Contextualización del problema de la obesidad. Obesidad Un Enfoque Multidisciplinario, 201-216.

Julia, A., Milian, G., David, E., \& García, C. (2016). La obesidad como factor de riesgo , sus determinantes y tratamiento Obesity as a risk factor, its determinants and treatment. $R e$ vista Cubana de Medicina General Integral. 2016; 32(3) ARTÍCULO, 32(3), 1-13.

Kalligeros, M., Shehadeh, F., Mylona, E. K., Benitez, G., Beckwith, C. G., Chan, P. A., \& Mylonakis, E. (2020). Association of Obesity with Disease Severity Among Patients with Coronavirus Disease 2019. Obesity, 28(7), 1200-1204. https://doi.org/10.1002/oby.22859

Kim, H. S. (2021). Do an altered gut microbiota and an associated leaky gut affect COVID-19 severity? MBio, 12(1), 1-9. https://doi.org/10.1128/mBio.03022-20

Klang, E., Kassim, G., Soffer, S., Freeman, R., Levin, M. A., \& Reich, D. L. (2020). Severe Obesity as an Independent Risk Factor for COVID-19 Mortality in Hospitalized Patients Younger than 50. Obesity, 28(9), 1595-1599. https://doi.org/10.1002/oby.22913

Pajuelo-Ramírez, J. (2017). La obesidad en el Perú TT - Obesity in Peru. Anales de La Facultad de Medicina, 78(2), 179-185. https://doi.org/10.15381/anales.v78i2.13214

Palomino-Pérez, A. M. (2020). Rol de la emoción en la conducta alimentaria. Revista Chilena de Nutrición, 47(2), 286-291. https://doi.org/10.4067/s0717-75182020000200286

Pérez B. (2014). Salud: entre la actividad física y el sedentarismo. An Venez Nutr [internet] 2014 [citado el 2 de mayo del 2019]; 27(1): [119-128]. 27(1), 119-128. http://bvs.minsa.gob.pe/local/MINSA/3299.pdf\%0Ahttp://www.scielo.org.ve/scielo. php?script=sci_arttext\&pid=S0798-07522014000100017\&lang=pt\%0Ahttp://www.scielo. org.ve/pdf/avn/v27n1/art17.pdf 
Quirantes Moreno, A. J., López Ramírez, M., Hernández Meléndez, E., \& Pérez Sánchez, A. (2009). Estilo de vida, desarrollo científico-técnico y obesidad. Revista Cubana de Salud Publica, 35(3), 1-8. https://doi.org/10.1590/s0864-34662009000300014

Rodríguez Ponciano, M. G. (2020). Adicciones en la época de la COVID-19. Unam, 1(3), 4.

Ryan, D. H., Ravussin, E., \& Heymsfield, S. (2020). COVID 19 and the Patient with Obesity The Editors Speak Out. Obesity, 28(5), 847. https://doi.org/10.1002/oby.22808

Salle, U. La, \& Ayala-moreno, M. R. (2020). Cambios en el comportamiento alimentario en la era del COVID-19. Relais, 3(1), 27-30.

Tenorio-Mucha, J., \& Hurtado-Roca, Y. (2020). Revisión sobre obesidad como factor de riesgo para mortalidad por COVID-19. Acta Medica Peruana, 37(3), 324-329. https://doi.org/10.35663/amp.2020.373.1197

Yeoh, Y. K., Zuo, T., Lui, C. Y., Zhang, F., Liu, Q., Li, A. Y., Chung, A. C., Cheung, C. P., Tso, E. Y., Fung, K. S., Chan, V., Ling, L., Joynt, G., Hui, D. S., Ng, S. S., Li, T. C., Ng, R. W., Yip, T. C., Wong, G., Chan F. K. ... Ng, S. C. (2021). Gut microbiota composition reflects disease severity and dysfunctional immune responses in patients with COVID-19. Gut, 1-9. https://doi.org/10.1136/gutjnl-2020-323020

WHO. Obesity: preventing and managing the global epidemic. Report of a WHO Consultation. WHO Technical Report Series 894. Geneva: World Health, (2000). https://www.who.int/nutrition/publications/obesity/WHO_TRS_894/en/ 